Compton effect with non-relativistic kinematics

This content has been downloaded from IOPscience. Please scroll down to see the full text. 2011 Phys. Educ. 46538

(http://iopscience.iop.org/0031-9120/46/5/003)

View the table of contents for this issue, or go to the journal homepage for more

Download details:

IP Address: 14.139.155.11

This content was downloaded on 29/03/2016 at 08:32

Please note that terms and conditions apply. 


\title{
Compton effect with non-relativistic kinematics
}

\author{
T Shivalingaswamy ${ }^{1,2}$ and B A Kagali ${ }^{2,3}$ \\ ${ }^{1}$ Department of Physics, Government College For Women, Mandya-571401, India \\ ${ }^{2}$ Department of Physics, Bangalore University, Bangalore-560056, India \\ E-mail: bakagali@gmail.com
}

\begin{abstract}
The change in wavelength of photons scattered by atomic electrons is deduced using non-relativistic kinematics, giving suitable justification.
\end{abstract}

\section{Introduction}

In deducing the change of wavelength of $x$ rays scattered by atomic electrons, one normally makes use of relativistic kinematics for electrons. However, recoiling energies of the electrons are of the order of a few $\mathrm{keV}$ which is less than $0.2 \%$ of their rest energies. Hence we may ask whether relativistic formulae are really necessary. Here we derive the formula for the change in wavelength of $\mathrm{x}$-rays using non-relativistic kinematics for electrons. It so happens, by some coincidence, that relativistic kinematics leads to exact simplification of the equations. Perhaps that is the reason for its use rather than physical considerations [1-3].

\section{Energy and momentum conservation equations}

In Compton scattering, an incident photon of frequency $v$ is supposed to collide with an electron at rest. The scattered photon is thought to have frequency $v^{\prime}<v$. The angle of scattering is taken as $\theta$ for the photon while the angle for the recoiling electron is taken as $\phi$. Both the angles are with respect to the direction of the incident photon, as shown in figure 1.

Considering the momentum and energy of the incident photon to be $\frac{h v}{c}$ and $h v$, respectively, and treating the electron as a non-relativistic particle,

\footnotetext{
3 Author to whom any correspondence should be addressed.
}

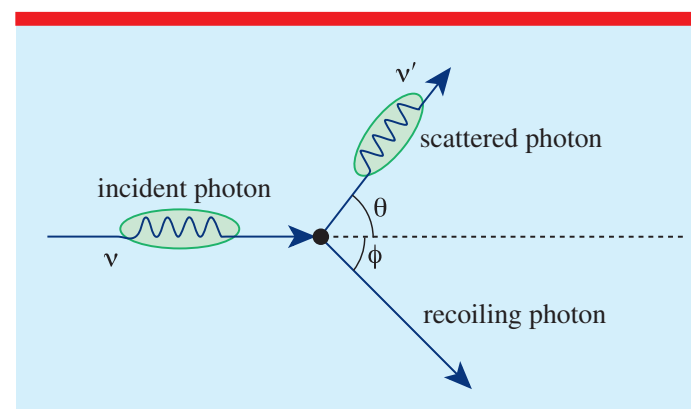

Figure 1. Angles involved in Compton scattering.

we get the following energy and momentum conservation equations.

Conservation of energy yields

$$
h v=h v^{\prime}+\frac{1}{2} m u^{2} .
$$

Conservation of momentum along the incident photon direction gives us

$$
\frac{h v}{c}=\frac{h v^{\prime}}{c} \cos \theta+m u \cos \phi .
$$

Conservation of momentum in the transverse direction to the incident photon leads to

$$
0=\frac{h v^{\prime}}{c} \sin \theta+m u \sin \phi .
$$

Eliminating $\phi$ from (2) and (3),

$$
\left(\frac{h v}{c}-\frac{h v^{\prime}}{c} \cos \theta\right)^{2}+\left(\frac{h v^{\prime}}{c}\right)^{2} \sin ^{2} \theta=m^{2} u^{2} .
$$




\section{Compton effect with non-relativistic kinematics}

This can be simplified to

$$
\left(\frac{h v}{c}\right)^{2}-2 \frac{h v}{c} \frac{h v^{\prime}}{c} \cos \theta+\left(\frac{h v^{\prime}}{c}\right)^{2}=m^{2} u^{2}
$$

Eliminating $u^{2}$ with the help of equation (1), we get

$$
\begin{aligned}
& \left(\frac{h v}{c}\right)^{2}-2 \frac{h v}{c} \frac{h v^{\prime}}{c} \cos \theta+\left(\frac{h v^{\prime}}{c}\right)^{2} \\
& =2 m h\left(v-v^{\prime}\right) .
\end{aligned}
$$

As the change in wavelength or frequency is less than $5 \%$, putting $v^{\prime}=v-\delta v$ and retaining only the first power of $\delta \nu$, the left-hand side (LHS) of equation (6) can be written as

$$
\begin{aligned}
\text { LHS } & =\left(\frac{h v}{c}\right)^{2}-2 \frac{h v}{c}\left(\frac{h}{c}\right)(v-\delta v) \cos \theta \\
& +\frac{h^{2}}{c^{2}}\left(v^{2}-2 v \delta v\right) .
\end{aligned}
$$

Simplifying this we get

$$
\begin{aligned}
\text { LHS } & =2\left(\frac{h v}{c}\right)^{2}-2\left(\frac{h v}{c}\right)^{2} \cos \theta \\
& -2 \frac{h^{2}}{c^{2}} \nu \delta v(1-\cos \theta) .
\end{aligned}
$$

Further simplification of this equation leads to

$$
\text { LHS }=2 \frac{h^{2} v}{c^{2}}(v-\delta v)(1-\cos \theta) .
$$

Since $v-\delta v=v^{\prime}$, we get

$$
\mathrm{LHS} \cong 2\left(\frac{h^{2} v v^{\prime}}{c^{2}}\right)(1-\cos \theta) .
$$

Equating equation (8) to the right-hand side (RHS) of equation (6), we get

$$
2\left(\frac{h^{2} v v^{\prime}}{c^{2}}\right)(1-\cos \theta) \cong 2 m h\left(v-v^{\prime}\right) .
$$

Dividing this by $2 m h$ we get the equation

$$
v-v^{\prime} \cong \frac{h v v^{\prime}}{m c^{2}}(1-\cos \theta) \text {. }
$$

In terms of wavelengths this can be written as

$$
\lambda^{\prime}-\lambda \cong \frac{h}{m c}(1-\cos \theta) .
$$

This is the standard Compton effect equation.

\section{Results and discussion}

With the physical parameters existing in Compton scattering it is not inappropriate to use the nonrelativistic kinematics for electrons as we have shown above.

Consider a collision in which the incident photon imparts maximum energy to the electron and thus it is backscattered [4]. Therefore, the maximum change in wavelength $\Delta \lambda=\lambda^{\prime}-\lambda=$ $\frac{2 h}{m c}=0.04852 \AA$.

Taking the wavelength of incident $\mathrm{x}$-rays to be $1 \times 10^{-10} \mathrm{~m}$, the percentage change in wavelength is $4.852 \%$, which is in fact equal to the percentage change in energy of the photon. A photon of wavelength $1 \times 10^{-10} \mathrm{~m}$ carries energy of $12.41 \mathrm{keV}$. Therefore the decrease in the energy of the photon is $0.6 \mathrm{keV}$. This energy lost by the photon appears as the recoiling energy of the electron. This kinetic energy is very much less than the rest energy of the electron, which is about $511 \mathrm{keV}$. Hence it is sufficient to use nonrelativistic kinematics for electrons rather than relativistic. We hope this short article clears the myth that relativistic kinematics is a must in Compton scattering.

Received 22 December 2010, in final form 1 April 2011 doi:10.1088/0031-9120/46/5/003

\section{References}

[1] Landau L D and Lifshitz E M 1998 Non-Relativistic Quantum Mechanics (Oxford: Butterworth-Heinmann)

[2] Englert B G (ed) 2001 Julian Schwinger Quantum Mechanics-Symbolism of Atomic Measurements (Berlin: Springer)

[3] Capri A Z 2002 Relativistic Quantum Mechanics and Introduction to Quantum Field Theory (Singapore: World Scientific)

[4] Bransden B H and Joachain C J 2004 Quantum Mechanics 2nd edn, First Indian reprint (Delhi: Pearson Education) 